\title{
The entanglement structure of quantum field systems
}

\author{
Vincent Lam*†
}

\begin{abstract}
This essay considers the question of the ontology of relativistic quantum field theory (RQFT). It aims to discuss possible implications of the peculiarities of quantum entanglement and quantum non-locality within the algebraic approach to RQFT. Fundamental results such as the Reeh-Schlieder theorem do not only show that quantum entanglement is a truly central feature of RQFT, but also that quantum entanglement displays specific features that are absent at the level of quantum mechanics (QM). Although these technical results are well-known, their possible implications for the ontology of the theory have been surprisingly little discussed. In particular, I will consider how the board ontic structural realist understanding of quantum entanglement and quantum non-locality within QM naturally applies to RQFT and can contribute to the debate on the ontology of the theory. More generally, this essay aims to illustrate the relevance of the peculiar status of quantum entanglement and quantum non-locality within RQFT for the ontological debate.
\end{abstract}

Keywords: relativistic quantum field theory, algebraic approach, ontology, quantum fields, quantum entanglement, Reeh-Schlieder theorem, non-separability, ontic structural realism

\section{Introduction}

Relativistic quantum field theory (RQFT) is currently our best fundamental physical theory about matter and constitutes the theoretical background of the experimentally successful Standard Model of high energy physics. In a scientific realist perspective, RQFT is therefore a natural candidate for ontological investigations about fundamental features of the physical world.

Much of the current discussion on the ontology of RQFT revolves around the debate between particle and field interpretations of the theory. Whereas it is widely recognized that standard particle interpretations face huge difficulties within RQFT, the (possibly field-theoretic) ontology of the theory remains unclear.

This essay suggests considering the question of the ontology of RQFT from a different angle. It does not aim to provide a complete ontology for RQFT. Its goal is more modest and more specific: it aims to discuss possible implications of the peculiarities of quantum entanglement and quantum non-locality within (the algebraic approach to) RQFT. Fundamental results such as the Reeh-Schlieder theorem do not only show that quantum entanglement is a truly central feature of RQFT, but also that quantum entanglement displays specific features that are absent at the level of quantum mechanics (QM). Although these technical results are well-known, their possible implications for the ontology of the theory have been surprisingly little discussed. In particular, I will consider how the broad ontic structural realist (OSR) understanding of quantum entanglement and quantum non-locality within QM naturally applies to RQFT and

*Department of Philosophy, University of Lausanne, CH-1015 Lausanne, Switzerand and School of History, Philosophy, Religion and Classics \& School of Mathematics and Physics, University of Queensland, QLD 4072, Australia

${ }^{\dagger}$ I am grateful to the Australian Research Council (ARC, Discovery Early Career Researcher Award (DECRA), project DE120102308) and to the Swiss National Science Foundation (SNSF, Ambizione grant PZ00P1_142536/1) for financial support. 
can contribute to the debate on the ontology of the theory. More generally, this essay aims to illustrate the relevance of the peculiar status of quantum entanglement and quantum non-locality within RQFT for the ontological debate.

\section{Algebraic tools}

The algebraic approach to quantum field theory (AQFT) provides a mathematically rigorous and conceptually clear framework that facilitates the discussion of foundational issues. Indeed, powerful technical results about entanglement have been obtained within AQFT. This section introduces the basic notions needed for the discussion of entanglement within AQFT.

With this focus on the algebraic approach, no particular stance is taken against the relevance of other variants of RQFT such as conventional, standard textbook ('cutoff' or 'renormalized') RQFT for foundational and interpretative issues (see Wallace 2006, 2011 and Fraser 2009, 2011 for two main positions in this debate). The algebraic framework provides tools for discussing precise results about entanglement, some of which actually remain relevant within the other variants.

Within AQFT, one associates to each bounded open spacetime region $O$ a $C^{*}$-algebra $\mathcal{A}(O)$, whose self-adjoint elements represent the observables in $O$, such that certain physically meaningful conditions (about the causal structure of spacetime, relativistic covariance and some aspects of locality among others) are satisfied (there is no need here to state these conditions explicitly, a classical exposure can be found in Haag 1996, §III.1; more recently see Halvorson 2006, $\S 2)$. Each $\mathcal{A}(O)$ can be understood as a $C^{*}$-subalgebra of a $C^{*}$-algebra $\mathcal{A}(\equiv \mathcal{A}(M))$. Within this framework and in a first approach, the fundamental entities described by the theory are naturally considered to be spacetime regions instantiating quantum field-theoretic properties, ${ }^{1}$ which are encoded in the algebraic structures associated with these regions - these fundamental entities described by local $C^{*}$-algebras are called 'quantum field systems' (however, as discussed below, quantum field systems need not be spacetime regions, see section 7).

One can define the state of a quantum field system over a spacetime region $O$ as a normalized positive linear functional $\omega$ on $\mathcal{A}(O)$ into the complex numbers, providing the expectation value in the state $\omega$ for each element of $\mathcal{A}(O)$. The link with the standard QM (i.e. Hilbert space) way of thinking is given by some relevant representation on a Hilbert space $\mathcal{H}_{\omega}$ (defined by some state $\omega$ for $\mathcal{A}$ via the Gelfand-Naimark-Segal (GNS) construction), which allows one to associate with each $\mathcal{A}(O)$ a (von Neumann) subalgebra $\mathcal{R}(O)$ of the algebra $\mathcal{B}\left(\mathcal{H}_{\omega}\right)$ of bounded linear operators on $\mathcal{H}_{\omega}$. The GNS theorem ensures that such representation $\pi_{\omega}: \mathcal{A} \rightarrow \mathcal{B}\left(\mathcal{H}_{\omega}\right)$ exists for a given state $\omega$ and that there exists a vector $\left|\Omega_{\omega}\right\rangle \in \mathcal{H}_{\omega}$ such that $\omega(A)=\left\langle\Omega_{\omega}\left|\pi_{\omega}(A)\right| \Omega_{\omega}\right\rangle, \forall A \in \mathcal{A}$; in the infinite-dimensional cases, two different states $\omega$ and $\omega^{\prime}$ may give rise to unitary inequivalent representations, which lead to some of the core interpretative problems of RQFT, see the recent monograph Ruetsche (2011).

\section{Reeh-Schlieder theorem and generic entanglement}

The formalism of AQFT provides the relevant framework to highlight a fundamental result about entanglement, the Reeh-Schlieder theorem. Let us consider a GNS representation with respect to some global state $\omega$, with local algebras acting on the Hilbert space $\mathcal{H}_{\omega}$, which possesses some Poincaré-invariant vacuum state $|\Omega\rangle$. The Reeh-Schlieder theorem basically says that the action of operators associated with any bounded open spacetime region $O$ on the vacuum state $|\Omega\rangle$ in $\mathcal{H}_{\omega}$ allows one to get arbitrarily close (in norm) to any state in $\mathcal{H}_{\omega}(|\Omega\rangle$ is said to be 'cyclic' for any local algebra of operators - this is true for any bounded energy state). In particular, the action on the vacuum of operators localized in $O$, which can be arbitrarily small, can generate

\footnotetext{
${ }^{1}$ How exactly these fundamental properties might account for what we experience (e.g. definite measurement outcomes) also depends on the strategy one adopts with respect to the measurement problem, see section 8 .
} 
(in the above sense) any state departing arbitrarily from the vacuum for regions that are spacelike separated from $O$. Most importantly, this surprising result finds its roots in the fact that the vacuum state is indeed a highly entangled state (Redhead 1995). ${ }^{2}$

Let $\mathcal{R}\left(O_{A}\right), \mathcal{R}\left(O_{B}\right) \subseteq \mathcal{B}\left(\mathcal{H}_{w}\right)$ be mutually commuting non-abelian (von Neumann) algebras of operators on $\mathcal{H}_{w}$ associated with space-like separated bounded spacetime regions $O_{A}$ and $O_{B}$. If $|\Psi\rangle$ is cyclic for $\mathcal{R}\left(O_{A}\right)$ (or $\mathcal{R}\left(O_{B}\right)$ ), then the induced algebraic state $\omega_{\Psi}$ on the von Neumann algebra $\mathcal{R}_{A B}=\left[\mathcal{R}\left(O_{A}\right) \cup \mathcal{R}\left(O_{B}\right)\right]^{\prime \prime}$ generated by $\left(\mathcal{R}\left(O_{A}\right), \mathcal{R}\left(O_{B}\right)\right)$ is entangled across $\left(O_{A}, O_{B}\right)$ (the commutant $\mathcal{R}^{\prime}$ is the algebra of operators that commute with every element of $\mathcal{R}$ and taking the double commutant ensures that the algebra is closed under the weak operator topology). A state of $\mathcal{R}_{A B}$ is entangled across $\left(O_{A}, O_{B}\right)$ if one cannot get arbitrarily close (in the weak operator topology) to it with convex combinations of product states of $\mathcal{R}_{A B}$ (see Clifton and Halvorson 2001 and references therein for the details - the rough presentation here of the AQFT results about entanglement mainly relies on their account). So, from the Reeh-Schlieder theorem we see that the vacuum $|\Omega\rangle$ is actually entangled across any space-like separated regions (and this is valid for all bounded energy states). More generally, from the Reeh-Schlieder theorem, one has that a (norm) dense set of states on $\mathcal{R}_{A B}$ are entangled across $\left(O_{A}, O_{B}\right)$, whenever $O_{A}$, $O_{B}$ are space-like separated bounded open spacetime regions. In this sense, space-like separated quantum field systems are said to be generically entangled (even if one considers non-interacting, free quantum fields). ${ }^{3}$

\section{More entanglement}

The AQFT framework allows one to cast further light in this context on the specific features of quantum entanglement that are absent at the QM level with finitely many degrees of freedom. Without entering into the many technical details, further properties of the physically relevant algebras within AQFT (the fact that the physically relevant algebras are all 'type III factors') imply that there cannot even be product states across any such algebra $\mathcal{R} \subseteq \mathcal{B}\left(\mathcal{H}_{w}\right)$ and its commutant $\mathcal{R}^{\prime} \subseteq \mathcal{B}\left(\mathcal{H}_{w}\right)$. In particular, we have in these cases that any global state is entangled across any diamond or double cone spacetime region $\diamond$ and its causal complement $\diamond^{\prime}$ (since for diamond regions the duality relation $\mathcal{R}\left(\diamond^{\prime}\right)=\mathcal{R}(\diamond)^{\prime}$ holds), where $\diamond$ between $p_{1}$ and $p_{2}\left(p_{2}\right.$ in the future light cone of $p_{1}$ ) is the intersection between the future light cone of $p_{1}$ and the past light cone of $p_{2}$ and where $\diamond^{\prime}$ is the set of points that are space-like separated from those of $\diamond$. The quantum field systems corresponding to these basic diamond regions are 'intrinsically' entangled with their environment understood in the sense of their spacelike complement.

The fact that in these cases there are no product states across $\left(\mathcal{R}, \mathcal{R}^{\prime}\right)$ lies at the basis of the claim that quantum entanglement is more entrenched and more robust in AQFT than in QM. The mathematical ground resides in the fact that the types of algebras involved in QM and AQFT are radically different ('type I' and 'type III' respectively; the main difference being the presence or absence of abelian, minimal, finite projections). In very rough terms, a state over a type III $C^{*}$-algebra is 'intrinsically mixed' in the sense that it cannot be represented as a density operator within the GNS representation defined by any pure state (the folia defined by the intrinsically mixed states are all disjoint from every folium defined by pure states). As a consequence - and this is the important point - such intrinsically mixed state cannot be understood as a probability distribution over pure states (one-dimensional projections), as in the case of density operators (which are always available in QM). Clifton and Halvorson (2001) argue that the very distinction between proper and improper mixtures then becomes "irrelevant"; more precisely, it seems to me that we are rather left only with improper mixtures in the sense of mixtures arising from entanglement (indeed, since intrinsically mixed states cannot be understood as probability distributions over pure states, it seems that there is no

\footnotetext{
${ }^{2}$ From an operational point of view, one can make sense of cyclicity by highlighting the need of selective operations in the considered region $O$ (Clifton and Halvorson 2001, 17-19).

${ }^{3}$ Strictly speaking, such generic entanglement is not specific to the quantum field-theoretic domain (Clifton and Halvorson 2001, 21-23).
} 
room for proper mixtures in such cases). Such states may clearly pose some difficulties to the standard interpretations of QM, in particular those relying on some epistemic (ignorance) understanding of the quantum state (Ruetsche 2004, 2011). ${ }^{4}$ The important point for this essay is that physically relevant cases within AQFT (such as a Klein-Gordon field in Minkwoski spacetime) involve intrinsically mixed states and these latter highlight how fundamental within AQFT quantum entanglement is (there are no pure states for 'type III factors') and encode in a precise sense how quantum entanglement is more fundamental in AQFT than in QM (where there always are pure states).

There is yet a further important aspect in which entanglement is more robust within AQFT: quantum field systems generically violate Bell-type inequalities (e.g. all states across $\left(\mathcal{R}, \mathcal{R}^{\prime}\right)$ above violate Bell-type inequalities), with generic maximal violation in many unexpected cases, such as non-interacting quantum field systems defined on diamond regions and their causal complement (Summers and Werner 1987, Halvorson and Clifton 2000). Beyond the issue of the exact meaning of quantum entanglement, which actually also depends on the interpretative strategy that is adopted with respect to the measurement problem (see section 8), the violation of Bell-type inequalities - which is experimentally verified in the QM context - is widely understood as a consequence of some fundamental quantum non-locality. From this point of view, the just mentioned generic maximal violation means that non-locality is more entrenched in AQFT than in QM.

It should be clear that these conclusions about entanglement are reached using the rigorous tools of AQFT. However, they can be more broadly understood as a consequence of the very quantum field-theoretic nature of the cases under consideration - in the sense of involving infinite number of degrees of freedom - and so can be considered relevant within RQFT in general.

\section{$5 \quad$ Entanglement and non-separability}

In this section and the next, I discuss how quantum entanglement (and more generally quantum non-locality) can be convincingly interpreted in structuralist terms within QM. Then, in the last section before the conclusion, I will discuss possible implications of such a structuralist interpretation for the ontology of (the algebraic approach to) RQFT, taking into account the peculiar status of entanglement in this theory.

To the extent that the physical states of a quantum system encode its physical properties, entangled quantum systems (with respect to certain degrees of freedom) cannot be said to possess (the corresponding) definite intrinsic state-dependent properties determined by their own, distinct state. A fortiori, the state (and the relevant properties) of the total system (composed by the entangled systems) are not determined by (do not supervene on) the 'states' (as given by the relevant reduced density operators, since no definite vectors in the relevant Hilbert space can be assigned) of the entangled systems.

It has been argued that this peculiar (strong) ${ }^{5}$ failure of supervenience is best understood in terms of non-separability in the sense that each entangled system does not possess any distinct independent state on their own (and so, to the extent that physical states describe physical properties, does not possess state-dependent properties on their own) and consequently the state (and the relevant properties) of the composed system are not determined by the distinct states (properties) of the systems (Teller 1986, Howard 1989, Healey 1991, Esfeld 2004).

The physical properties described by the physical states may typically take some probabilistic form within quantum theory. Physical states can therefore be directly characterized in terms of relevant (conditional) probability distributions. If probabilities are understood in some realist, objective way (the exact details of what that could mean are not needed here), the mere fact that joint probability distributions for composite systems do not factorize into

\footnotetext{
${ }^{4}$ It is rather surprising that such fact, which can be fruitful for fundamental interpretative issues in quantum theory, does not seem to have been widely exploited yet. Such discussion would obviously require separate treatment.

${ }^{5}$ See Cleland (1984).
} 
the product of independent distributions for single subsystems vindicate the just described nonseparability picture. Moreover, Howard (1989) and Shimony (1989), among others, argue that the experimentally verified violation of Bell-type inequalities is best understood in terms of non-separability, which is encoded in the violation of the outcome independence hypothesis ${ }^{6}$. Together with the parameter independence condition ${ }^{7}$, the outcome independence condition is equivalent to the so-called 'Bell locality' condition or factorization condition (Jarrett 1984), from which Bell-type inequalities can be rather straightforwardly derived (together with some less debatable assumptions).

The main difficulty of (and possible objection against) non-separability is that it seems hard to make sense of it. Indeed, the standard conception of physical entities or objects (in a broad sense) requires that they have to be something in themselves, possess some intrinsic identity independently of other physical entities, and so be described by some distinct independent physical state. As a consequence, non-separable entities are hard to conceive within such framework. This standard conception is still deeply entrenched (even if implicitly) in the contemporary thinking about nature. For instance, in his book on the philosophy of QM, Dickson explicitly argues against any rejection of what he presents as a version of Einstein's position, according to which "the state of a single particle can be completely characterized without reference to any other" $(1998,155)$. The argument is that if we reject such assumption (about separability), then it seems that we cannot even refer to physical entities in the first place since we don't really have objects that are something by themselves.

\section{Ontic structural realism}

OSR provides a conceptual framework where non-separability does make sense and more generally where quantum entanglement (and quantum non-locality) is taken seriously at the ontological level. I take OSR to be an ontological conception about what there is in the world, namely physical structures in the sense of networks of concrete, physical relations among entities ('relata') whose existence (and possible identity) depends on the relations they enter into (and on the structures they are part of). ${ }^{8}$ According to this view, OSR only aims to provide a convincing interpretation of given fundamental physical theories (here, quantum theory); it does not aim to provide any argument in the debate about scientific realism, some sufficiently strong version of which is here merely taken as a working hypothesis (OSR can be understood as form of scientific realism). ${ }^{9}$

Within quantum theory, OSR is most widely discussed in connection with the permutation invariance of similar elementary quantum particles; in particular their possible weak discernibility and its link with OSR have been recently much debated (Saunders 2006, Dieks and Versteegh 2008, Muller and Saunders 2008, Muller and Seevinck 2009, Ladyman and Bigaj 2010, Ainsworth 2011). Whereas weak discernibility clearly offers a favorable environment for OSR (Muller 2011), this latter conception is not committed to weak discernibility or to the validity of any version of Leibniz's Principle of the Identity of Indiscernibles (PII) (Ainsworth 2011): nothing in the central claim of OSR as presented above implies any commitment to discernibility or the PII.

I now want to emphasize that OSR also provides an interpretative framework for understanding quantum entanglement (and non-locality). ${ }^{10}$ Indeed, OSR allows one to make sense

\footnotetext{
${ }^{6}$ The terminology is Shimony's, where outcome independence is understood as the stochastic independence of the measurement outcomes on one entangled system from the outcomes on the other systems (we consider the case of two systems for simplicity); outcome independence can be convincingly interpreted as a separability condition, although it has not to be so, see Fogel (2007); for a critical view, see Maudlin (2011, 85-90).

${ }^{7}$ Stochastic independence of the measurement outcomes on one system from the measurement settings on the other.

${ }^{8}$ Note that this characterization of OSR does not entail that there are no relata or no objects, only that they cannot exist and have any identity independently of one another.

${ }^{9}$ This is a more restricted aim than within the original French and Ladyman's OSR, which also explicitly aims to account for theory change (Ladyman 1998, French and Ladyman 2003, French 2006).

${ }^{10}$ The cases of similar elementary quantum particles and of quantum entanglement are related but clearly distinct
} 
of quantum non-separability in terms of the lack of intrinsic identity - lack of independent existence if one prefers to avoid talk about identity - of the considered entangled physical systems. Such entangled systems do not possess any distinct independent physical state and any (statedependent) properties on their own; they can be ascribed neither (state-dependent) properties independently of the systems they are entangled with nor identity conditions that distinguish them from the systems they are entangled with. As a consequence, they do not possess any intrinsic identity (they do not exist independently of one another).

Even without intrinsic identity and independent existence, entangled quantum systems can however be genuinely considered to stand in quantum entanglement relations, which are understood as concrete physical relations such as the relation 'having opposite direction of each component of spin to' in the famous singlet case. Quantum entanglement relations can also be concretely described as correlations among single system probability distributions (the explicit expression of these correlations of course depends of the case under consideration), which can be understood in terms of relational properties of the entangled subsystems (if one accepts probabilistic properties as genuine ones - the argument here does however not rely on such commitment, insofar as quantum entanglement can still be considered as a concrete physical relation). ${ }^{11}$ As a consequence, even if lacking intrinsic identity and independent existence, entangled, non-separable quantum systems can be understood as genuine physical entities in the sense that they bear the physical relations of quantum entanglement to one another (these physical relations and relational properties can be predicated to them $)^{12}$.

Moreover, quantum entanglement relations are genuine irreducible physical relations in the sense that they are not determined by properties of the entangled systems - they are (strongly) non-supervenient - but only by the properties (state) of the total composed system. The picture we obtain is therefore the one suggested by OSR, that of a network of fundamental physical relations (quantum entanglement relations) ${ }^{13}$ among physical relata (fundamental quantum systems) that do not possess any qualitative intrinsic identity (and any independent existence) over and above the relations in which they stand. Their numerical identity makes only sense structurally, that is in terms of the physical relations they stand in, and this is the case independently of whether their numerical identity is considered to be grounded in some weak version of the PII or simply to be a primitive notion (because of the arguable lack of meaningful identity conditions): in the former case, numerical identity is grounded in the weakly discernible relations and in the latter case, primitive numerical identity is structural or contextual in the sense that it is not intrinsic and not equivalent to haecceitism (Ladyman 2007). I take this claim about the structural identity and interdependent existence of fundamental entangled quantum systems to be one of the main morals of QM, which OSR aims to encode.

\section{Ontological aspects of quantum field theory}

There is an interesting sense in which the algebraic approach to RQFT encodes the understanding of entanglement in terms of non-separability, therefore providing a hospitable environment

for the point of view of OSR; the latter case has been far less discussed within the framework of OSR, see Esfeld (2004) and Ladyman et al. (2007, §3.4).

${ }^{11}$ We don't really need the distinction between relational properties and relations insofar as the considered physical relations give rise to the relata having physical relational properties and the other way round. This latter relationship between relations and relational properties should however be reminded in order to avoid some confusion about the ontological commitment of OSR: contrary to what Ainsworth (2010) claims, OSR is not committed to saying that entangled quantum systems have no properties.

${ }^{12}$ There is in any case a precise sense in which entangled quantum systems can be meaningfully considered to stand in (possibly weakly discernible) relation, see the careful discussion in Muller and Saunders (2008); therefore the 'third problem' for OSR in Ainsworth $(2010,54)$ is clearly avoided.

${ }^{13}$ As mentioned above (section 4) and further discussed below (section 8), the exact nature of the quantum entanglement relations also depends on the interpretative strategy with respect to the measurement problem. More broadly, one can here consider relations of quantum non-locality, which feature in all (realist) interpretations to the extent that these latter all have to account for the experimentally verified violation of Bell-type inequalities. 
for OSR along the lines discussed in the previous section. Indeed, parameter independence is actually encoded in the very locality (local action) condition ('axiom') of AQFT, so that the (generic maximal) violation of Bell inequalities within the AQFT framework directly 'implies' the violation of outcome independence (Butterfield 1995). To the extent that such a violation of outcome independence can be genuinely interpreted in terms of non-separability (see above section 5), the AQFT framework involves generic non-separability among quantum field systems.

Quantum field systems are non-separable in the sense that the joint probabilities (expectation values) are not determined by the marginals (as given by the states over the relevant algebras). To the extent that these probabilities reflect properties of the corresponding quantum field systems, these latter cannot be ascribed any intrinsic identity (that is, independently of other quantum field systems) on their basis. The point here is completely analogous to the one in the QM case, and the OSR understanding of entanglement within QM can naturally be applied to entanglement within (the algebraic approach to) RQFT.

The peculiar status of quantum entanglement (and quantum non-locality) within AQFT discussed above in sections 3 and 4 further strengthens this point and may reveal interesting novel aspects of the ontology of the RQFT domain. It suggests that entanglement (non-locality) is a universal feature of physical quantum field systems in the sense that all quantum field systems (including free, non-interacting ones) satisfying certain physically reasonable conditions tend to be ultimately entangled (in particular with their environment understood as their causal complement). This consideration suggests in turn to consider the conceptual framework offered by OSR as a general one applying to all quantum fields - again, to the extent that they are all ultimately entangled.

Before discussing some possible implications of the OSR understanding of entanglement for the ontology of (the algebraic approach to) RQFT, two points need to be clarified. First, the above claim about the generic and even universal character of entanglement within (the algebraic approach to) RQFT seems at odds with the obvious existence, for instance, of basic non-entangled $n$-particle states within the Fock space representation for a free system. This consideration is about the scope of entanglement within RQFT and does not constitute any objection to the OSR interpretation of entanglement. The proponent of AQFT would point out that considering non-entangled states within a specific representation has only limited meaning since it does not take into account the existence of unitary inequivalent representations (which constitutes one of the main interpretative problems of RQFT, and which AQFT precisely aims to take into account). ${ }^{14}$ Of course, in many practical situations, it is effectively relevant to consider non-entangled states for quantum field systems that are, strictly speaking, entangled.

This last consideration leads us to the second point. If entanglement and non-separability are universal and generic in the above sense, then one may wonder how physics on local systems can be done without taking into account the whole universe at once. ${ }^{15}$ It seems that the possibility of some meaningful physical analysis is tied to the possibility to consider fundamental physical systems independently of one another in some concrete operational sense. The rather surprising fact is that, despite the fundamental and generic character of entanglement in the theory, such possibility is precisely available in AQFT. Indeed, the very algebraic structure of quantum fieldtheoretic observables encodes several notions of operational independence - the most important one being the split property ${ }^{16}$ - that ensure the operational good behaviour of quantum field systems. ${ }^{17}$

\footnotetext{
${ }^{14}$ French (2012) interestingly suggests various ways to account for the existence of unitary inequivalent representations within the interpretative framework of OSR.

${ }^{15}$ Conceptual and methodological worries about quantum entanglement and non-separability were most famously expressed by Einstein (1948, 321-322), quoted and translated in Howard (1985, 187-188).

${ }^{16}$ For a pair of (von Neumann) algebras $\left(\mathcal{R}\left(O_{A}\right), \mathcal{R}\left(O_{B}\right)\right.$ ), the split property is basically equivalent to the existence of an isomorphism between the algebra $\mathcal{R}_{A B}$ they generate and the (von Neumann algebra) tensor product of $\mathcal{R}\left(O_{A}\right)$ and $\mathcal{R}\left(O_{B}\right)$. In particular, this fact implies the existence of a product state on $\mathcal{R}_{A B}$.

${ }^{17}$ The split property ensures that quantum field systems can be independently and locally prepared in arbitrary states. The split property is typically satisfied in operationally interesting cases, e.g. for strictly space-like separated quantum field systems (see the discussion in Clifton and Halvorson 2001, 28-29 and Summers 2009).
} 
Now, from the OSR understanding of generic entanglement in (the algebraic approach to) RQFT, what can be said about the ontology of the theory? Given the difficulties of the standard particle interpretation (Halvorson and Clifton 2002, Fraser 2008), ${ }^{18}$ it is widely (sometimes tacitly) acknowledged that RQFT is about quantum fields (or quantum field systems). How exactly these latter should be understood remains however controversial. One the one hand, as mentioned above in section 2, the algebraic approach to RQFT naturally suggests to understand quantum field systems in terms of spacetime regions instantiating quantum field-theoretic properties, which are mathematically represented by the algebraic structures (including the state) associated with these regions (Wallace and Timpson 2010, $\S 5$ recently defend such a view). On the other hand, such an interpretation relies on a classical background spacetime that lies outside the scope of (standard) RQFT. But the general theory relativity (GTR) teaches us that spacetime is itself a dynamical entity, encoding the gravitational field, which is expected to ultimately possess a quantum nature as well. These considerations suggest to understand quantum field systems, possibly including quantum gravitational field systems, as fundamental substantival entities on their own, not reducible to spacetime regions instantiating quantum field-theoretic properties. For instance, within the framework of AQFT, the structure of $C^{*}$-algebras representing the quantum field systems can be understood as primitive, and the topological, differential and metrical structures representing spacetime can then be derived from the algebraic structure (Dieks 2001, §12-13 articulates and defends such a view) ${ }^{19}$.

It has been argued that these two distinct interpretations of quantum field systems (in terms of spacetime regions or as fundamental substantival entities on their own) constitute a case of metaphysical underdetermination, which can be dissolved by adopting OSR (French and Ladyman 2003, §6). Besides the fact that it is not clear how OSR would concretely dissolve such alleged underdetermination, I consider that the point here is ultimately to be settled empirically. For instance, if a fully background independent quantum field theory of gravitation could be elaborated and shown to be experimentally successful, then it would vindicate the second interpretation, since there would be no background spacetime regions to act as bearers of quantum field-theoretic properties.

The OSR understanding of generic entanglement in the algebraic approach to RQFT offers a different perspective on the ontology of the theory. Within this framework, quantum field systems, whatever their ultimate nature is, are primarily understood as interdependent physical entities (relata) embedded in a network of irreducible fundamental physical relations entanglement relations. In other words, the ontology of (the algebraic approach to) RQFT is an ontology of 'entanglement structures', understood in the sense of networks of entanglement relations among quantum field systems whose existence - what it means for a quantum field system to be the one it is - depends on the entanglement relations they enter into (on the structure they are part of)..$^{20}$

\section{Open perspectives}

Of course no complete and coherent ontology of RQFT can be given without addressing the quantum measurement problem, and moving from QM to RQFT does not help at all in that respect (see e.g. the discussion in Barrett 2002). The precise ontological meaning of quantum entanglement actually depends on the approach to the measurement problem that is favored. However, it does not imply that ontological investigations at the RQFT level - 'beyond' standard discussions around the measurement problem within QM - are meaningless. Quite the contrary indeed: any ontology that takes RQFT seriously has to take into account the peculiarities of quantum field-theoretic entanglement and non-locality described above (using algebraic tools). In particular, as mentioned in section 4, within the algebraic approach, intrinsic entanglement

\footnotetext{
${ }^{18}$ Bain (2010) offers an alternative point of view on these difficulties.

${ }^{19}$ The trope bundle interpretation of AQFT proposed in Kuhlmann (2010) also goes along these lines to some extent.

${ }^{20}$ French (2010) discusses the different notions of ontological dependence that are relevant in the context of OSR.
} 
and intrinsically mixed states may well constitute an important and interesting challenge for the standard interpretations of quantum theory (i.e. for the standard solutions to the measurement problem).

OSR provides an interpretative and ontological framework for understanding entangled quantum field systems that highlights their structural features in the sense of their ontological interdependence (lack of independent existence and lack of intrinsic identity in particular). These structural features are independent of the different (realist) approaches to the measurement problem to the extent that these latter - Bohmian, dynamical collapse (GRW) or Everett-type approaches - have to account for the peculiar features of quantum field-theoretic entanglement and non-locality (e.g. generic maximal violation of Bell-type inequalities).$^{21}$

Furthermore, ontological investigations at the RQFT level, such as the one proposed in this essay, may be relevant for two fundamental open issues. First, they may allow to provide some elements of a broad interpretative and ontological framework for the possible quantum nature of the gravitational field (and of spacetime itself), as described for instance by the canonical quantization programs of GTR. In other words, these investigations may be helpful for developing an ontological picture that remains relevant in the quantum gravitational context. Indeed, quantum correlations seem to constitute a fundamental feature of the quantum gravitational domain, at least as described by the canonical approaches, such as standard quantum geometrodynamics where generic entanglement among various types of degrees of freedom at the cosmological scale is expected to produce some relevant decoherence effects - or loop quantum gravity - where, strictly speaking, the quantum gravitational state corresponding to an arbitrary region of space is actually an entangled state (a 'mixed spin network state').

Second, and more specifically, ontological investigations in the context of AQFT allows one to explicitly take into account the existence of unitary inequivalent representations. French (2012) has recently argued that OSR provides a powerful interpretative framework for these inequivalent representations, where symmetry relations and "inter-representational" relations - relations that "cut across different representations" - play a fundamental role. Of course, it would be of great interest for the OSR project (and for the ontology of RQFT) to make explicitly the link between the concrete (physical) structural features highlighted in this essay and these seemingly more formal (mathematical) structural features. A lot of work remains to be done, but the prospects of OSR as a convincing ontological framework for RQFT are good.

\footnotetext{
${ }^{21}$ An important and difficult project would be to further substantiate this claim by explicit investigations of the different (realist) approaches to the measurement problem in the RQFT domain, e.g. Bohmian RQFT, dynamical collapse RQFT and Everett RQFT. Contrary to what Esfeld (2012) suggests, the fact that OSR provides a general ontological framework which remains genuine and can be further specified within these different quantum interpretations does constitute a positive and convincing feature of this conception. It actually provides strong evidence that OSR captures genuine essential ontological features of the world as described by current fundamental physics.
} 


\section{References}

P. M. Ainsworth. What is ontic structural realism? Studies in History and Philosophy of Modern Physics, 41:50-57, 2010.

P. M. Ainsworth. Ontic structural realism and the principle of the identity of indiscernibles. Erkenntnis, 75:67-84, 2011.

J. Bain. Quantum field theories in classical spacetimes and particles. Studies in History and Philosophy of Modern Physics, 42:98-106, 2010.

J. A. Barrett. On the Nature of Measurement Records in Relativistic Quantum Field Theory. In M. Kuhlmann, H. Lyre, and A. Wayne, editors, Ontological Aspects of Quantum Field Theory. World Scientific, Singapore, 2002.

J. Butterfield. Vacuum Correlations and Outcome Dependence in Algebraic Quantum Field Theory. Annals of the New York Academy of Sciences, 755:768-785, 1995.

C. Cleland. Space: An abstract system of non-supervenient relations. Philosophical Studies, 46: 19-40, 1984.

R. Clifton and H. Halvorson. Entanglement and open systems in algebraic quantum field theory. Studies in History and Philosophy of Modern Physics, 32:1-31, 2001.

W. M. Dickson. Quantum Chance and Non-locality. Cambridge University Press, Cambridge, 1998.

D. Dieks. Space and Time in Particle and Field Physics. Studies in History and Philosophy of Modern Physics, 32(2):217-241, 2001.

D. Dieks and M. A. M. Versteegh. Identical quantum particles and weak discernibility. Foundations of Physics, 38:923-934, 2008.

A. Einstein. Quanten-Mechanik und Wirklichkeit. Dialectica, 2:320-324, 1948.

M. Esfeld. Quantum entanglement and a metaphysics of relations. Studies in History and Philosophy of Modern Physics, 35:601-617, 2004.

M. Esfeld. Ontic structural realism and the interpretation of quantum mechanics. European Journal for the Philosophy of Science, DOI 10.1007/s13194-012-0054-x, 2012.

B. Fogel. Formalizing the separability condition in Bell's theorem. Studies in History and Philosophy of Modern Physics, 38:920-937, 2007.

D. Fraser. The fate of 'Particles' in quantum field theory with interactions. Studies in History and Philosophy of Modern Physics, 39:841-859, 2008.

D. Fraser. Quantum Field Theory: Underdetermination, Inconsistency, and Idealization. Philosophy of Science, 76:536-567, 2009.

D. Fraser. How to take particle physics seriously: A further defence of axiomatic quantum field theory. Studies in History and Philosophy of Modern Physics, 42:126-135, 2011.

S. French. Structure as a weapon of the realist. Proceedings of the Aristotelian Society, 106: 169-187, 2006.

S. French. The interdependence of structure, objects and dependence. Synthese, 175:89-109, 2010. 
S. French. Unitary inequivalence as a problem for structural realism. Studies in History and Philosophy of Modern Physics, doi:10.1016/j.shpsb.2011.09.00, 2012.

S. French and J. Ladyman. Remodelling Structural Realism: Quantum Physics and the Metaphysics of Structure. Synthese, 136(1):31-56, 2003.

R. Haag. Local Quantum Physics. Springer, New York (2nd ed.), 1996.

H. Halvorson. Algebraic Quantum Field Theory. In J. Butterfield and J. Earman, editors, Handbook of the Philosophy of Science. Vol. 2: Philosophy of Physics. Elsevier, Amsterdam, 2006.

H. Halvorson and R. Clifton. Generic Bell correlation between arbitrary local algebras in quantum field theory. Jounral of Mathematical Physics, 41(4):1711-1717, 2000.

H. Halvorson and R. Clifton. No Place for Particles in Relativistic Quantum Theories? Philosophy of Science, 69:1-28, 2002.

R. Healey. Holism and nonseparability. Jounral of Philosophy, 88:393-421, 1991.

D. Howard. Einstein on locality and separability. Studies in History and Philosophy of Science, 16:171-201, 1985.

D. Howard. Holism, Separability, and the Metaphysical Implications of the Bell Experiments. In J. Cushing and E. McMullin, editors, Philosophical Consequences of Quantum Theory: Reflections on Bells Theorem, pages 224-253. University of Notre Dame Press, Notre Dame, 1989 .

J. Jarrett. On the physical significance of the locality conditions in the Bell arguments. Noûs, 18:569-589, 1984.

M. Kuhlmann. The Ultimate Constituents of the Material World. Ontos Verlag, Heusenstamm, 2010.

J. Ladyman. What is structural realism? Studies in History and Philosophy of Science Part A, 29(3):409-424, 1998.

J. Ladyman. On the Identity and Diversity of Objects in a Structure. Proceedings of the Aristotelian Society Supplementary Volume, 81(1):45-61, 2007.

J. Ladyman and T. Bigaj. The Principle of the Identity of Indiscernibles and Quantum Mechanics. Philosophy of Science, 77:117-136, 2010.

J. Ladyman, D. Ross, D. Spurett, and J. Collier. Every Thing Must Go: Metaphysics Naturalized. Oxford University Press, Oxford, 2007.

T. Maudlin. Quantum Non-Locality and Relativity. Wiley-Blackwell, Chichester, 3rd edition, 2011.

F. A. Muller. Withering away, weakly. Synthese, 180:223-233, 2011.

F. A. Muller and S. Saunders. Discerning Fermions. The British Journal for the Philosophy of Science, 59:499-548, 2008.

F. A. Muller and M. Seevinck. Discerning Elementary Particles. Philosophy of Science, 76: 179-200, 2009.

M.L.G Redhead. More Ado about Nothing. Foundations of Physics, 25, 1995. 
L. Ruetsche. Intrinsically mixed states: an appreciation. Studies in History and Philosophy of Modern Physics, 35:221-239, 2004.

L. Ruetsche. Interpreting Quantum Theories. Oxford University Press, Oxford, 2011.

S. Saunders. Are quantum particles objects? Analysis, 66:52-63, 2006.

A. Shimony. Search for a Worldview Which Can Accommodate Our Knowledge of Microphysics. In J.T. Cushing and E. McMullin, editors, Philosophical Consequences of Quantum Theory: Reflections on Bell's Theorem, pages 25-37. University of Notre Dame, Notre Dame, 1989.

S.J. Summers. Subsystems and independence in relativistic microscopic physics. Studies in History and Philosophy of Modern Physics, 40:133-141, 2009.

S.J. Summers and R.F. Werner. Maximal violation of Bell's inequalities is generic in quantum field theory. Communications in Mathematical Physics, 110:247-259, 1987.

P. Teller. Relational Holism and Quantum Mechanics. The British Journal for the Philosophy of Science, 37:275-307, 1986.

D. Wallace. In Defence of Naiveté: The Conceptual Status of Lagrangian Quantum Field Theory. Synthese, 151:33-80, 2006.

D. Wallace. Taking particle physics seriously: A critique of the algebraic approach to quantum field theory. Studies in History and Philosophy of Modern Physics, 42:116-125, 2011.

D. Wallace and G. T. Timpson. Quantum Mechanics on Spacetime I: Spacetime State Realism. The British Journal for the Philosophy of Science, 61(4):697-727, 2010. 